\title{
Fast-slow analysis for parametrically and externally excited systems with two slow rationally related excitation frequencies
}

\author{
Xiujing Han, ${ }^{1}$ Qinsheng $\mathrm{Bi}^{1,}{ }^{1 *}$ Peng $\mathrm{Ji},{ }^{2,3}$ and Jürgen Kurths ${ }^{2,3,4,5}$ \\ ${ }^{1}$ Faculty of Civil Engineering and Mechanics, Jiangsu University, Zhenjiang Jiangsu 212013, People's Republic of China \\ ${ }^{2}$ Potsdam Institute for Climate Impact Research, Potsdam 14473, Germany \\ ${ }^{3}$ Department of Physics, Humboldt University, Berlin 12489, Germany \\ ${ }^{4}$ Institute for Complex Systems and Mathematical Biology, University of Aberdeen, Aberdeen AB24 3UE, United Kingdom \\ ${ }^{5}$ Department of Control Theory, Nizhny Novgorod State University, Gagarin Avenue 23, 606950 Nizhny Novgorod, Russia
}

(Received 6 October 2014; revised manuscript received 18 December 2014; published 15 July 2015)

\begin{abstract}
We present a general method for analyzing mixed-mode oscillations (MMOs) in parametrically and externally excited systems with two low excitation frequencies (PEESTLEFs) for the case of arbitrary $m: n$ relation between the slow frequencies of excitations. The validity of the approach has been demonstrated using the equations of Duffing and van der Pol, separately. Our study shows that, by introducing a slow variable and finding the relation between the slow variable and the slow excitations, PEESTLEFs can be transformed into a fast-slow form with a single slow variable and therefore MMOs observed in PEESTLEFs can be understood by the classical machinery of fast subsystem analysis of the transformed fast-slow system.
\end{abstract}

DOI: 10.1103/PhysRevE.92.012911

PACS number(s): 05.45.-a, 82.40.Bj

\section{INTRODUCTION}

Many problems in physics, chemistry, biology, engineering, etc., can be described by nonlinear systems with slowly varying control parameters. From the view point of physics, this results from the fact that the results of a long-time experiment may depend on parameters that are varying slowly. For example, the weight of a rocket in flight slowly decreases due to the burning of fuel and improves the speed of the rocket $[1,2]$. Catalytic activities in chemical reactors slowly decline due to chemical erosion and decrease the reactor performance [3,4]. In all these problems, there are parameters that are varying slowly with time or are deliberately varied by the researcher. Slow linear simulation of a parameter is one of the most common hypotheses that describe the variety law of the slow parameter. Dynamical behaviors in such a situation have been intensively studied (see, e.g., [5-7]). Another interesting case is the slow periodic simulation of a parameter, i.e., the case when the control parameter is a slowly varying periodic function. Pisarchik et al. [8] provided experimental evidence that a slowly varying periodic parameter can control dynamical regimes and inhibit chaos in a nonlinear system. It has also been shown that a slowly varying periodic parameter can stabilize unstable orbits [9], annihilate one of the coexisting states, and thus results in controlled monostability [10]. In particular, a slowly varying periodic parameter can lead to periodic bifurcation delay behaviors, which have been identified as new routes to repetitive spiking and bursting [11]. However, most of the previous work focused on systems with a single slowly varying periodic parameter and there has been little work done on the analysis of dynamical systems with two slowly varying control parameters.

In this paper we use slow parametric and external excitations to simulate two slowly varying periodic parameters in

\footnotetext{
*biqinsheng@sohu.com
}

dynamical systems, governed by the general form

$$
\dot{x}=F\left(x, \beta_{1} \cos \left(\omega_{1} t\right)\right)+\beta_{2} \cos \left(\omega_{2} t\right),
$$

where $x \in \mathbb{R}^{n}$ is the vector of dynamics, $\beta_{1} \cos \left(\omega_{1} t\right)$ and $\beta_{2} \cos \left(\omega_{2} t\right)$ are the parametric and external excitations, respectively, and $\omega_{1}$ and $\omega_{2}$ are small $\left(\omega_{1} \ll 1\right.$ and $\left.\omega_{2} \ll 1\right)$, i.e., both of the excitations vary slowly, and denote slow cosinoidal modulations of two parameters in the dynamical system. We assume that the system (1) has only one time scale for the case when the two excitations are frozen, i.e., when $\omega_{1}=\omega_{2}=0$, there is no difference in the time scale among variables.

Numerous results for parametrically and externally excited systems play an important role in promoting the development of the theory of nonlinear dynamics and its application. Various qualitative and quantitative methods are used to study the response, bifurcations, and chaos in these systems (see, e.g., Refs. [12-18] and references therein). In this paper we give a different point of view on parametrically and externally excited systems when the two excitations vary slowly, i.e., from the viewpoint of fast-slow dynamics. Since the two excitations evolve on a relatively slow time scale, while the frozen system itself evolves on a relatively fast time scale, the system (1) belongs to a class of fast-slow systems with two slow variables. The fast subsystem is given by $\dot{x}=F\left(x, \beta_{1} \gamma_{1}\right)+\beta_{2} \gamma_{2}$, while the slow variables are $\cos \left(\omega_{1} t\right)$ and $\cos \left(\omega_{2} t\right)$.

Earlier work that treated a slowly varying excitation as a slow variable appeared in Ref. [19], where an unfolding theory approach to mixed-mode oscillations (MMOs) was investigated when the slow excitation traced a closed path in the universal unfolding of the singularity. The basic idea was presented in Ref. [20], where a classification scheme for MMOs was described. In Refs. [21,22] this idea was applied to the Duffing and van der Pol systems with a slowly varying external forcing term. Recently, it was extended to accommodate a van der Pol system with two slowly varying external forcing terms [23], where many complex MMOs were discovered.

Mixed-mode oscillations, also known as bursting oscillations, are complex oscillatory patterns characterized by a 

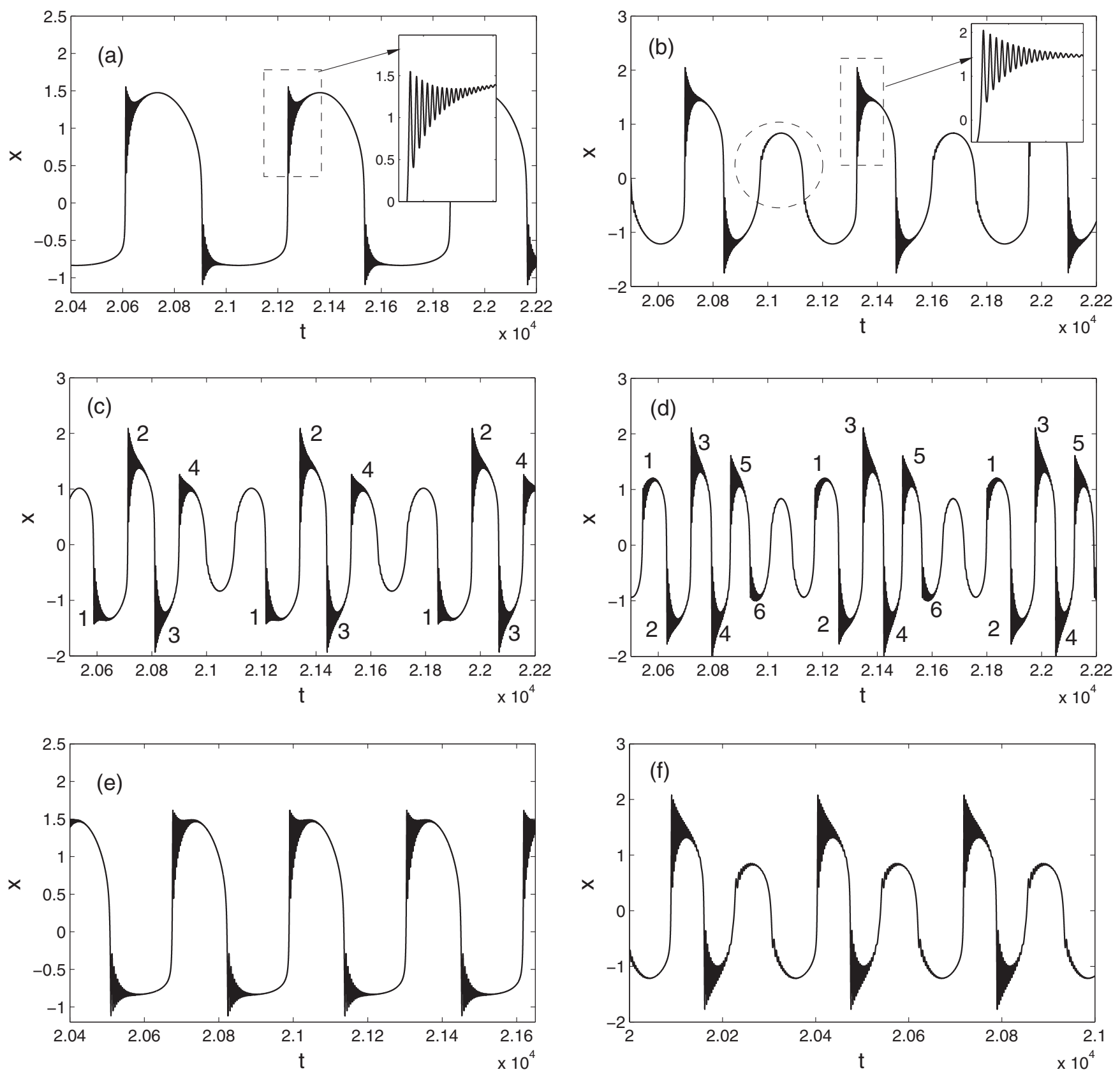

FIG. 1. Mixed-mode oscillations in the Duffing system (12) for (a) $\omega_{1}=\omega_{2}=0.01$, (b) $\omega_{1}=0.01$ and $\omega_{2}=0.02$, (c) $\omega_{1}=0.01$ and $\omega_{2}=0.03$, (d) $\omega_{1}=0.01$ and $\omega_{2}=0.04$, (e) $\omega_{1}=\omega_{2}=0.02$, and (f) $\omega_{1}=0.02$ and $\omega_{2}=0.04$. The insets in (a) and (b) are the enlargement of the rectangle areas and give a clear view of the oscillations. The circled region in (b) shows that the bursting in (b) exhibits different characteristics compared to the bursting in (a).

TABLE I. Parametrically and externally excited Duffing equation and the associated fast subsystem with different $\omega_{2}$ for fixed $\omega_{1}=0.01$.

\begin{tabular}{lccc}
\hline \hline$\omega_{1}$ & $\omega_{2}$ & Parametrically and externally excited Duffing equation & Fast subsystem \\
\hline 0.01 & 0.01 & $\ddot{x}+0.1 \dot{x}-[0.5+\cos (0.01 t)] x+x^{3}=\cos (0.01 t)$ & $\ddot{x}+0.1 \dot{x}-(0.5+\gamma) x+x^{3}=\gamma$ \\
0.01 & 0.02 & $\ddot{x}+0.1 \dot{x}-[0.5+\cos (0.01 t)] x+x^{3}=\cos (0.02 t)$ & $\ddot{x}+0.1 \dot{x}-(0.5+\gamma) x+x^{3}=2 \gamma^{2}-1$ \\
0.01 & 0.03 & $\ddot{x}+0.1 \dot{x}-[0.5+\cos (0.01 t)] x+x^{3}=\cos (0.03 t)$ & $\ddot{x}+0.1 \dot{x}-(0.5+\gamma) x+x^{3}=4 \gamma^{3}-3 \gamma$ \\
0.01 & 0.04 & $\ddot{x}+0.1 \dot{x}-[0.5+\cos (0.01 t)] x+x^{3}=\cos (0.04 t)$ & $\ddot{x}+0.1 \dot{x}-(0.5+\gamma) x+x^{3}=8 \gamma^{4}-8 \gamma^{2}+1$ \\
\hline
\end{tabular}



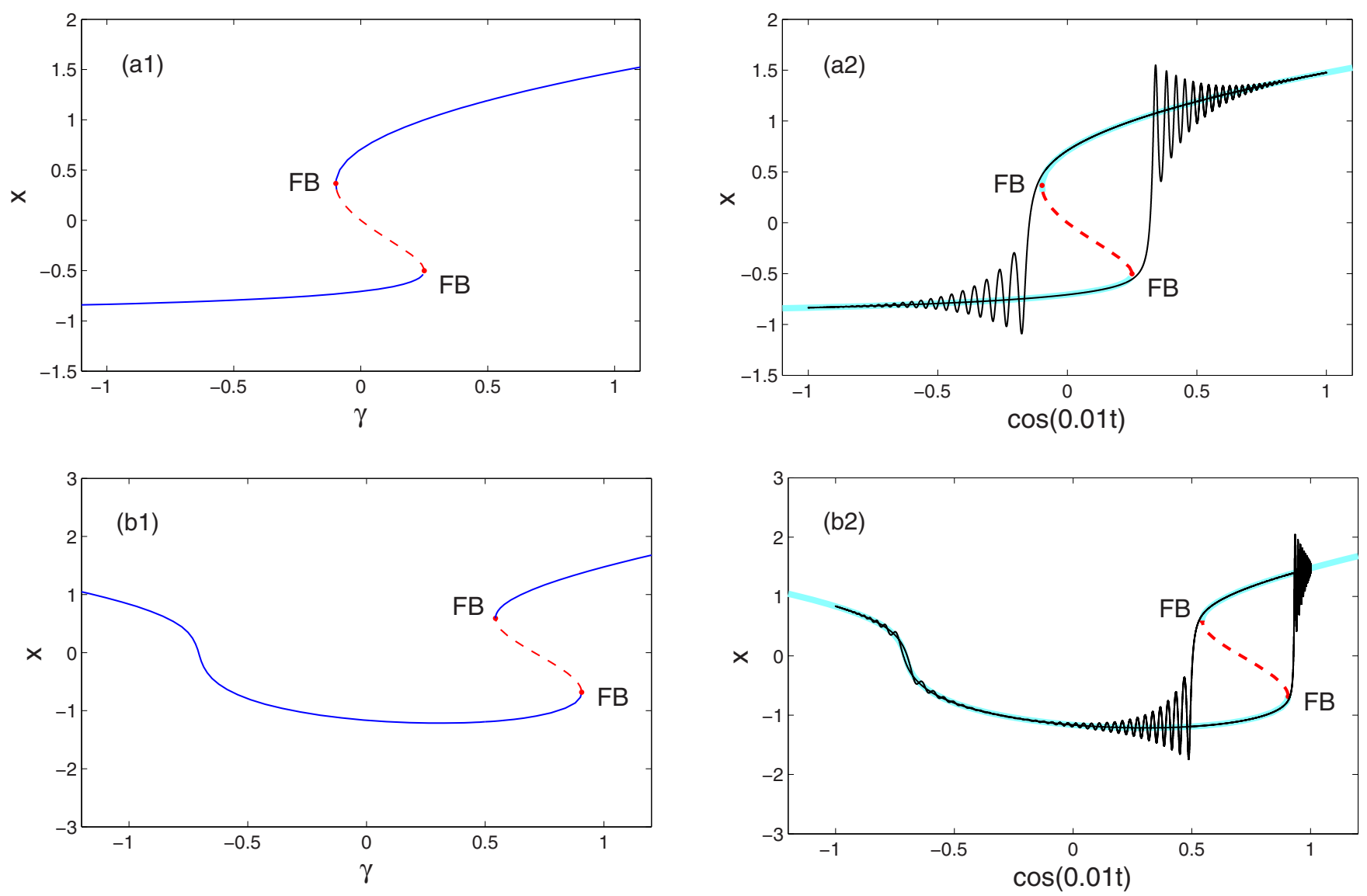

FIG. 2. (Color online) (a1) Bifurcation diagram of the fast subsystem (13), where $\gamma$ is used as the control parameter. Solid curves are stable equilibria, while the dashed curve indicates the unstable equilibrium. The FB denote fold bifurcations of the equilibria. (a2) Corresponding transformed phase diagram of MMOs, with the bifurcation diagram shown in (a1) superimposed, where the stable equilibria are highlighted with thick blue curves. (b1) and (b2) Same as in (a1) and (a2) for $\omega_{1}=0.01$ and $\omega_{2}=0.02$.

combination of small-amplitude and large-amplitude oscillations, which can be described by coupled fast and slow subsystems [24,25]. Rinzel [26] proposed the idea of using bifurcation analysis of a frozen or fast subsystem to explain MMO patterns. This idea is what we know as fast-slow analysis, which was applied to great effect in the work by Izhikevich [24] to create a classification of MMOs. Based on the fast-slow analysis, the underlying mechanisms of the appearance of MMOs in fast-slow systems with a single slow variable have been actively investigated [19-22,24-30].

Moreover, there is a vast literature on MMOs in systems with more than one slow variable. Rinzel [31] pointed out that to generate a parabolic MMO pattern [32] in an autonomous manner requires at least two slow variables. Izhikevich [24] reviewed MMOs in systems with two slow variables and presented some possible routes to MMOs. Milik et al. [33] and Krupa et al. [34] showed that, in fast-slow systems with two slow variables, the occurrence of MMOs is triggered by a generalized canard phenomenon and folded singularity. Curtu [35] and Guckenheimer [36] substantially showed that MMOs in fast-slow systems with two slow variables were also linked to singular Hopf bifurcations. Watts et al. [37] studied a type of bursting model with two slow variables, where the observed MMOs can be well understood by introducing a measure called the dominance factor. $\mathrm{Li}$ and $\mathrm{Bi}$ [38] investigated the mechanism of the generation of MMOs in a chemical reaction system with two slow variables. The repetitive spiking process of the MMOs is associated with weak excitation and can be understood by an enveloping slow-fast analysis. A detailed and thorough overview is presented in [39], where a survey of different types of MMOs concentrate the analysis on MMOs whose small-amplitude oscillations are produced by a local, multiple-time-scale mechanism.

Though much work has been done, a systematic and uniform method for analyzing MMOs in general fast-slow systems with more than one slow variable is still missing. In this paper we explore a general method for analyzing MMOs in a class of fast-slow systems with two slow variables, i.e., the parametrically and externally excited systems with two low excitation frequencies (PEESTLEFs), given by Eq. (1). The remainder of this paper is organized as follows. In Sec. II we provide a general method for analyzing MMOs in PEESTLEFs. In Sec. III we implement the method on Duffing and van der Pol equations to investigate MMOs. Dynamical mechanisms underlying the appearance of MMOs and their evolutions are investigated to validate the method. We show that PEESTLEFs exhibit rich and complex MMOs and our approach plays a key role in understanding such phenomena. Finally, in Sec. IV we conclude with a discussion of our results. 

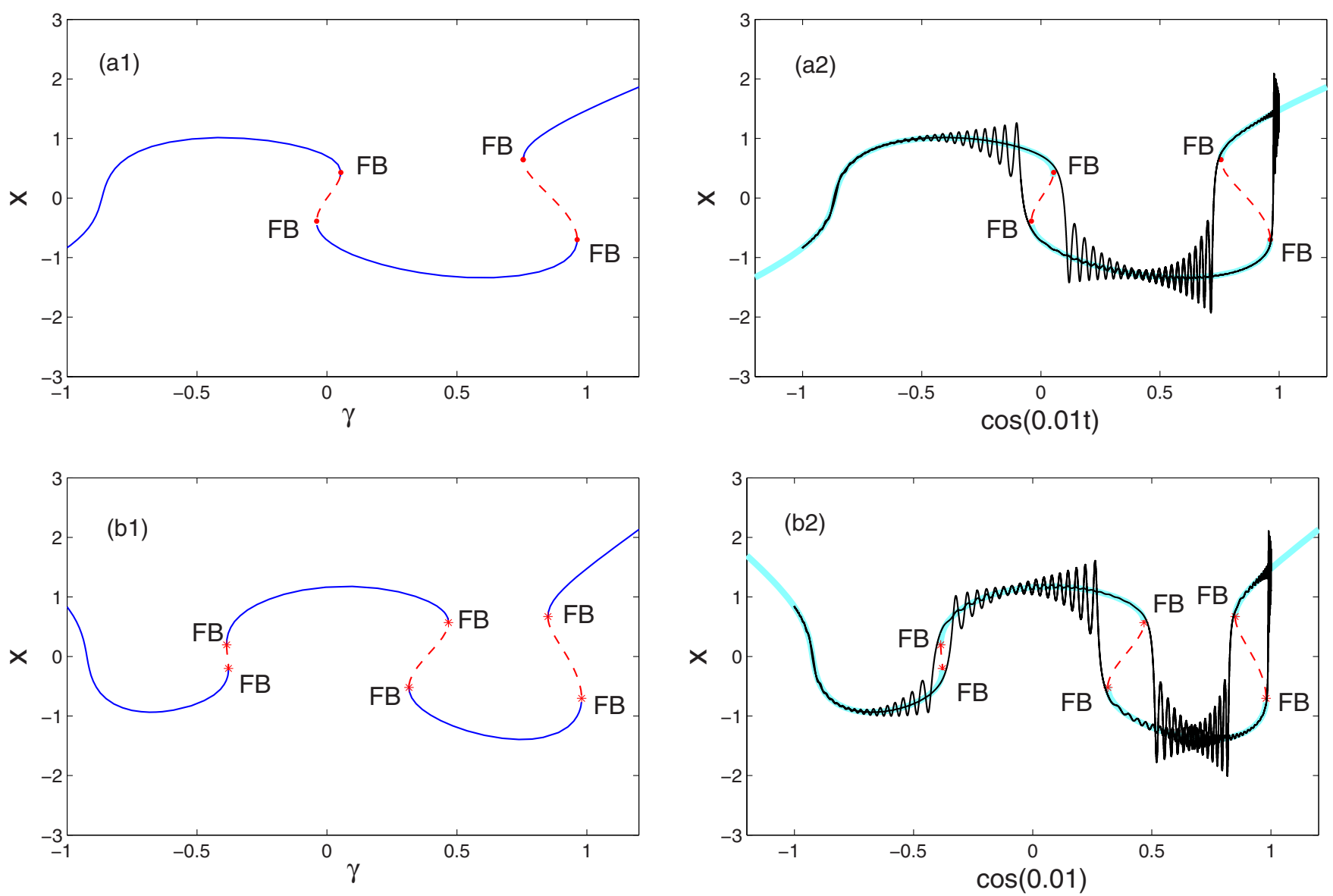

FIG. 3. (Color online) Same as in Fig. 2 for (a1) and (a2) $\omega_{1}=0.01$ and $\omega_{2}=0.03$ and (b1) and (b2) $\omega_{1}=0.01$ and $\omega_{2}=0.04$.

\section{GENERAL METHOD}

We start by outlining the method for analyzing MMOs in PEESTLEFs. The basic method is to transform PEESTLEFs, a class of fast-slow systems with two slow variables, into fastslow forms with a single slow variable. This is possible if we can find a slow variable $g(t)$ such that both $\cos \left(\omega_{1} t\right)$ and $\cos \left(\omega_{2} t\right)$ can be expressed as a function of $g(t)$, i.e., $\cos \left(\omega_{1} t\right)=$ $f_{1}(g(t))$ and $\cos \left(\omega_{2} t\right)=f_{2}(g(t))$. Then the PEESTLEFs can be rewritten in the form

$$
\dot{x}=F\left(x, \beta_{1} f_{1}(g(t))\right)+\beta_{2} f_{2}(g(t)),
$$

where $g(t)$ is the only slow variable. From a mathematical point of view, the transformed system (2) is no different compared to the original system (1). However, from the viewpoint of fastslow dynamics, the PEESTLEFs has been transformed into a fast-slow form with a single slow variable $g(t)$. Therefore,
MMOs observed in PEESTLEFs can be understood by the classic fast-slow analysis of the transformed system (2).

In addition, we find the slow variable $g(t)$ and the associated functions $f_{1}(x)$ and $f_{2}(x)$ according to the relation between the frequencies of the two excitations. To this end we consider the de Moivre formula [40]: For any real number $x$ and integer $n$, it holds that

$$
(\cos x+i \sin x)^{n}=\cos (n x)+i \sin (n x),
$$

where $i$ stands for the imaginary unit $\left(i^{2}=-1\right)$. Expanding the left-hand side and comparing the real and imaginary parts, one can derive expressions for $\cos (n x)$ and $\sin (n x)$. The expression for $\cos (n x)$ is given by

$$
\begin{aligned}
\cos (n x)= & C_{n}^{0} \cos ^{n} x+C_{n}^{2} \cos ^{(n-2)} x(i \sin x)^{2} \\
& +C_{n}^{4} \cos ^{(n-4)} x(i \sin x)^{4}+\cdots \\
& +C_{n}^{m} \cos ^{(n-m)} x(i \sin x)^{m},
\end{aligned}
$$

TABLE II. Parametrically and externally excited Duffing equation and the associated fast subsystem with different $\omega_{1}$ and $\omega_{2}$.

\begin{tabular}{cccc}
\hline \hline$\omega_{1}$ & $\omega_{2}$ & Parametrically and externally excited Duffing equation & Fast subsystem \\
\hline 0.02 & 0.01 & $\ddot{x}+0.1 \dot{x}-[0.5+\cos (0.02 t)] x+x^{3}=\cos (0.01 t)$ & $\ddot{x}+0.1 \dot{x}-\left[0.5+\left(2 \gamma^{2}-1\right)\right] x+x^{3}=\gamma$ \\
0.03 & 0.01 & $\ddot{x}+0.1 \dot{x}-[0.5+\cos (0.03 t)] x+x^{3}=\cos (0.01 t)$ & $\ddot{x}+0.1 \dot{x}-\left[0.5+\left(4 \gamma^{3}-3 \gamma\right)\right] x+x^{3}=\gamma$ \\
0.04 & 0.01 & $\ddot{x}+0.1 \dot{x}-[0.5+\cos (0.04 t)] x+x^{3}=\cos (0.01 t)$ & $\ddot{x}+0.1 \dot{x}-\left[0.5+\left(1+8 \gamma^{4}-8 \gamma^{2}\right)\right] x+x^{3}=\gamma$ \\
0.02 & 0.03 & $\ddot{x}+0.1 \dot{x}-[0.5+\cos (0.02 t)] x+x^{3}=\cos (0.03 t)$ & $\ddot{x}+0.1 \dot{x}-\left[0.5+\left(2 \gamma^{2}-1\right)\right] x+x^{3}=4 \gamma^{3}-3 \gamma$ \\
0.03 & 0.02 & $\ddot{x}+0.1 \dot{x}-[0.5+\cos (0.03 t)] x+x^{3}=\cos (0.02 t)$ & $\ddot{x}+0.1 \dot{x}-\left[0.5+\left(4 \gamma^{3}-3 \gamma\right)\right] x+x^{3}=2 \gamma^{2}-1$ \\
\hline \hline
\end{tabular}



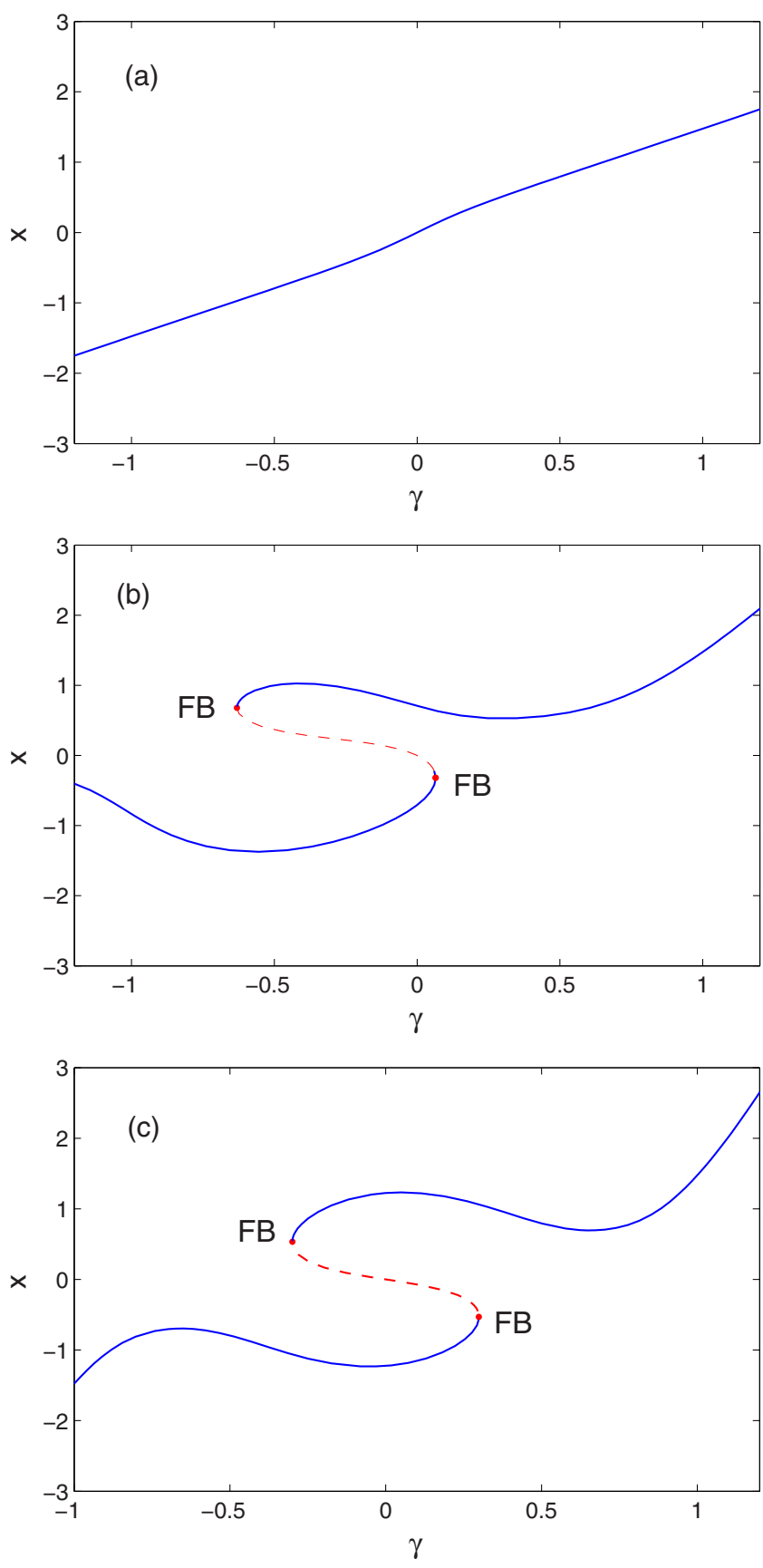

FIG. 4. (Color online) Bifurcation diagram of the fast subsystem for (a) $\omega_{1}=0.02$, (b) $\omega_{1}=0.03$, and (c) $\omega_{1}=0.04$. The frequency $\omega_{2}$ is fixed at $\omega_{2}=0.01$.

where $m(m \leqslant n)$ is the maximum even number no larger than $n$. Since the power of $i \sin (x)$ in each item of (4) is even and $\sin ^{2} x=1-\cos ^{2} x$, it is easy to check that $\cos (n x)$ can be expressed as a function of $\cos (x)$, i.e., $\cos (n x) \equiv f_{n}^{*}(\cos (x))$, where $f_{n}^{*}(x)$ follows the polynomial function

$$
\begin{aligned}
f_{n}^{*}(x)= & C_{n}^{0} x^{n}-C_{n}^{2} x^{n-2}\left(1-x^{2}\right)+C_{n}^{4} x^{n-4}\left(1-x^{2}\right)^{2} \\
& -\cdots+i^{m} C_{n}^{m} x^{n-m}\left(1-x^{2}\right)^{m / 2},
\end{aligned}
$$

where $m(m \leqslant n)$ is the maximum even number no larger than $n$.
Next let us explore the slow variable $g(t)$ and the functions $f_{1}(x)$ and $f_{2}(x)$ based on the above results. We consider the case of the arbitrary $m: n$ relation between the two frequencies of excitations $\left(\omega_{1}\right.$ and $\left.\omega_{2}\right)$.

Case A: $\frac{\omega_{1}}{\omega_{2}}=\frac{m}{n}$, where $m$ and $n$ are integers. There exists a largest integer $l$, i.e., the greatest common divisor of $m$ and $n$, that satisfies $m=p l$ and $n=q l$, where $p$ and $q$ are two prime numbers. Therefore, the slow frequencies can be written in the form $\omega_{1}=\varepsilon p l$ and $\omega_{2}=\varepsilon q l$ with $\varepsilon \ll 1$. Based on (4) and (5), we have $\cos \left(\omega_{1} t\right)=f_{p}^{*}(\cos (\varepsilon l t))$ and $\cos \left(\omega_{2} t\right)=$ $f_{q}^{*}(\cos (\varepsilon l t))$. So the slow variable $g(t)$ can be chosen as $g(t)=$ $\cos (\varepsilon l t)$ and the functions $f_{1}(x)$ and $f_{2}(x)$ can be selected as $f_{1}(x)=f_{p}^{*}(x)$ and $f_{2}(x)=f_{q}^{*}(x)$. Thus the PEESTLEFs can be written in a fast-slow form with a single slow variable, given by

$$
\dot{x}=F\left(x, \beta_{1} f_{p}^{*}(g(t))\right)+\beta_{2} f_{q}^{*}(g(t)),
$$

where $g(t)=\cos (\varepsilon l t)$ is the slow variable. Additionally, there are two special cases based on case A.

Case B: $\frac{\omega_{1}}{\omega_{2}}=n$, where $n$ is an integer. Since $\cos \left(\omega_{1} t\right)=$ $f_{n}^{*}\left(\cos \left(\omega_{2} t\right)\right)$, where $f_{n}^{*}(x)$ is the polynomial function given by (5), the slow variable $g(t)$ can be chosen as $g(t)=\cos \left(\omega_{2} t\right)$ and the functions $f_{1}(x)$ and $f_{2}(x)$ can be selected as $f_{1}(x)=f_{n}^{*}(x)$ and $f_{2}(x)=f_{1}^{*}(x)=x$, which yields

$$
\dot{x}=F\left(x, \beta_{1} f_{n}^{*}(g(t))\right)+\beta_{2} g(t),
$$

where $g(t)=\cos \left(\omega_{2} t\right)$ is the slow variable.

Case $C: \frac{\omega_{2}}{\omega_{1}}=n$, where $n$ is an integer. Since $\cos \left(\omega_{2} t\right)=$ $f_{n}^{*}\left(\cos \left(\omega_{1} t\right)\right)$, where $f_{n}^{*}(x)$ is given by (5), we have $g(t)=$ $\cos \left(\omega_{1} t\right), \quad f_{1}(x)=f_{1}^{*}(x)=x$, and $f_{2}(x)=f_{n}^{*}(x)$, which yields

$$
\dot{x}=F\left(x, \beta_{1} g(t)\right)+\beta_{2} f_{n}^{*}(g(t)),
$$

where $g(t)=\cos \left(\omega_{1} t\right)$ is the slow variable.

Bifurcation behavior of the fast subsystem plays an important role in the generation of MMOs. Traditionally, there are at least two bifurcations associated with MMOs and different MMOs can be obtained if the fast subsystem exhibits different bifurcation modes $[24,25]$. From Eqs. (6)-(8) one can see that the dynamic equation of the fast subsystem of PEESTLEFs changes with varying the frequencies of the two excitations. For example, for the fast-slow system (6), the associated fast subsystem is

$$
\dot{x}=F\left(x, \beta_{1} f_{p}^{*}(\gamma)\right)+\beta_{2} f_{q}^{*}(\gamma)
$$

for the system (7), the related fast subsystem is given by

$$
\dot{x}=F\left(x, \beta_{1} f_{n}^{*}(\gamma)\right)+\beta_{2} \gamma ;
$$

and for the system (8), the corresponding fast subsystem is

$$
\dot{x}=F\left(x, \beta_{1} \gamma\right)+\beta_{2} f_{n}^{*}(\gamma) .
$$

In Eqs. (9)-(11), $\gamma$ is the control parameter. Such changing of the dynamics of the fast subsystem of PEESTLEFs may further lead to different bifurcation modes, which provide essential conditions for the generation of different MMOs. Therefore, according to the above analysis, one can conclude 

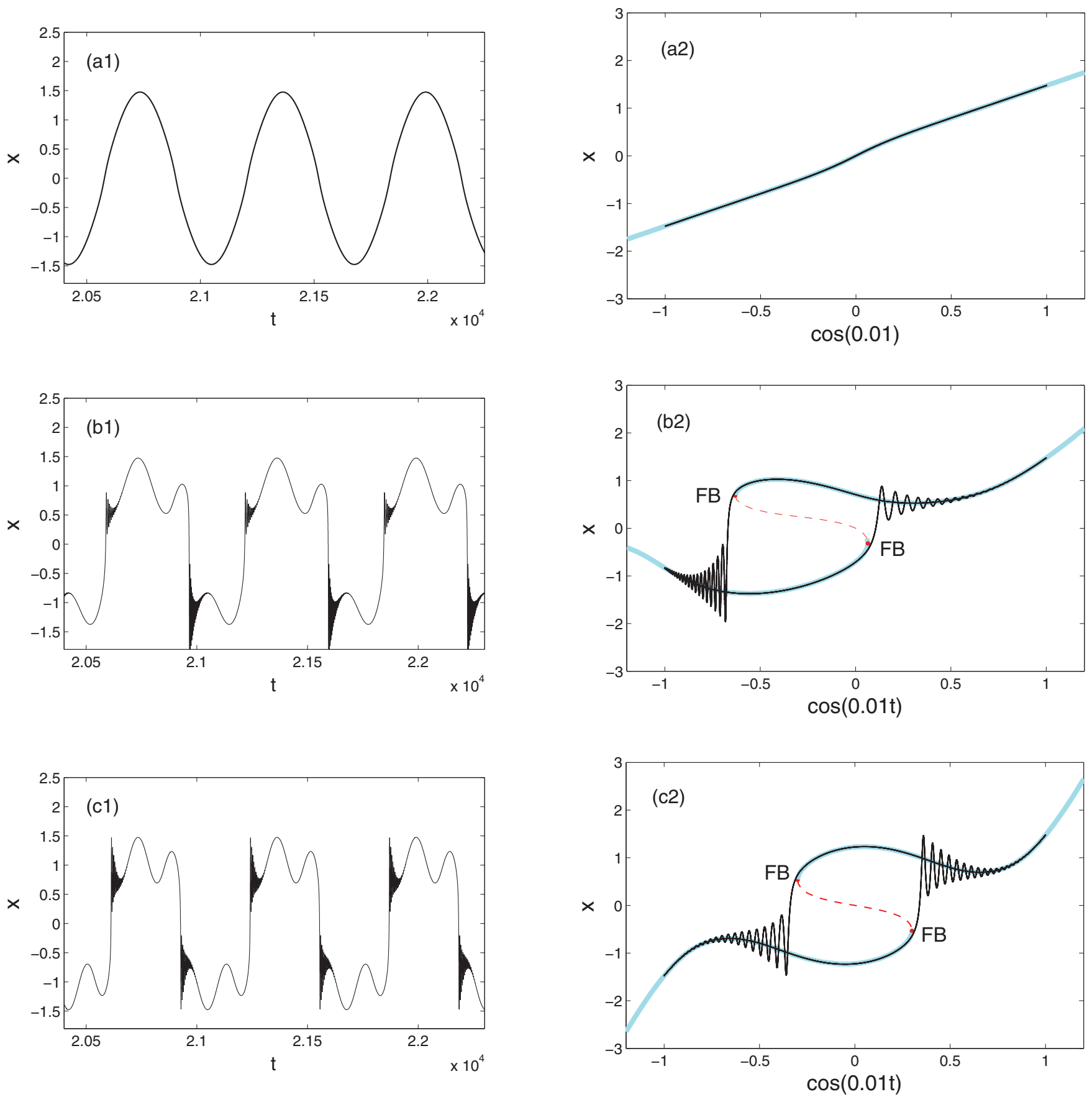

FIG. 5. (Color online) (a1) Time series of the solution for $\omega_{1}=0.02$ and $\omega_{2}=0.01$, (a2) transformed phase diagram of the solution and its overlay with the equilibrium curve in Fig. 4(a), (b1) and (b2) same as in (a1) and (a2) for $\omega_{1}=0.03$ and $\omega_{2}=0.01$, and (c1) and (c2) same as in (a1) and (a2) for $\omega_{1}=0.04$ and $\omega_{2}=0.01$. In (b2) and (c2) the FB indicate fold bifurcations of equilibria.

that, for PEESTLEFs, the generation of different MMOs is possible when we vary the frequencies of the two excitations.

Other factors to consider are the amplitudes $\beta_{1}$ and $\beta_{2}$. In the classical study of nonlinear oscillations, strong and weak excitations are considered separately, since the methods proposed to study systems with strong excitations may be quite different from those of systems with weak excitations, in particular in problems of nonlinear oscillators with weak and strong excitations $[41,42]$. However, from the viewpoint of fast-slow dynamics, strong and weak excitations are nothing special. The reason for this is that the slow variables are $\cos \left(\omega_{1} t\right)$ and $\cos \left(\omega_{2} t\right)$ and both amplitudes $\beta_{1}$ and $\beta_{2}$ belong to the fast subsystem, i.e., like other parameters in the fast subsystem, $\beta_{1}$ and $\beta_{2}$ are general parameters of the fast subsystem, e.g., see the fast subsystems (9)-(11). No matter what the magnitudes of the amplitudes are, the amplitudes can change qualitatively properties of the MMOs as long as they vary and pass through bifurcation points of the fast subsystem. 


\section{APPLICATIONS}

We have outlined our general method to analyze MMOs in PEESTLEFs. In what follows we validate the method for the equations of Duffing and van der Pol. We first give some typical examples of MMOs in Duffing and van der Pol equations for the case when the excitations vary slowly. Afterward, the dynamical mechanisms underlying the appearance of MMOs and their evolution will be studied by using the method.

\section{A. Duffing equation}

The parametrically and externally excited Duffing equation with two low excitation frequencies is given by

$$
\ddot{x}+\delta \dot{x}-\left[b+\beta_{1} \cos \left(\omega_{1} t\right)\right] x+x^{3}=\beta_{2} \cos \left(\omega_{2} t\right),
$$

where $0<\omega_{1,2} \ll 1$. In order to study the generation of MMOs, throughout this paper we fix $\delta=0.1, b=0.5$, and $\beta_{1}=\beta_{2}=1$. When the two excitations vary slowly, several interesting behaviors can be observed as follows: When $\omega_{1}=\omega_{2}=0.01$, a periodic MMOs pattern is exhibited [see Fig. 1(a)]; when $\omega_{1}=0.01$ and $\omega_{2}=0.02$, the MMOs show some different characteristics [see Fig. 1(b)]; with further increments of $\omega_{2}\left(\omega_{1}\right.$ is fixed at $\left.\omega_{1}=0.01\right)$, the MMOs vary and become complex [see Figs. 1(c) and 1(d)].

When $\omega_{1}=\omega_{2}=0.01$, since the two excitations have the same frequency, we treat $\cos (0.01 t)$ as the only slow
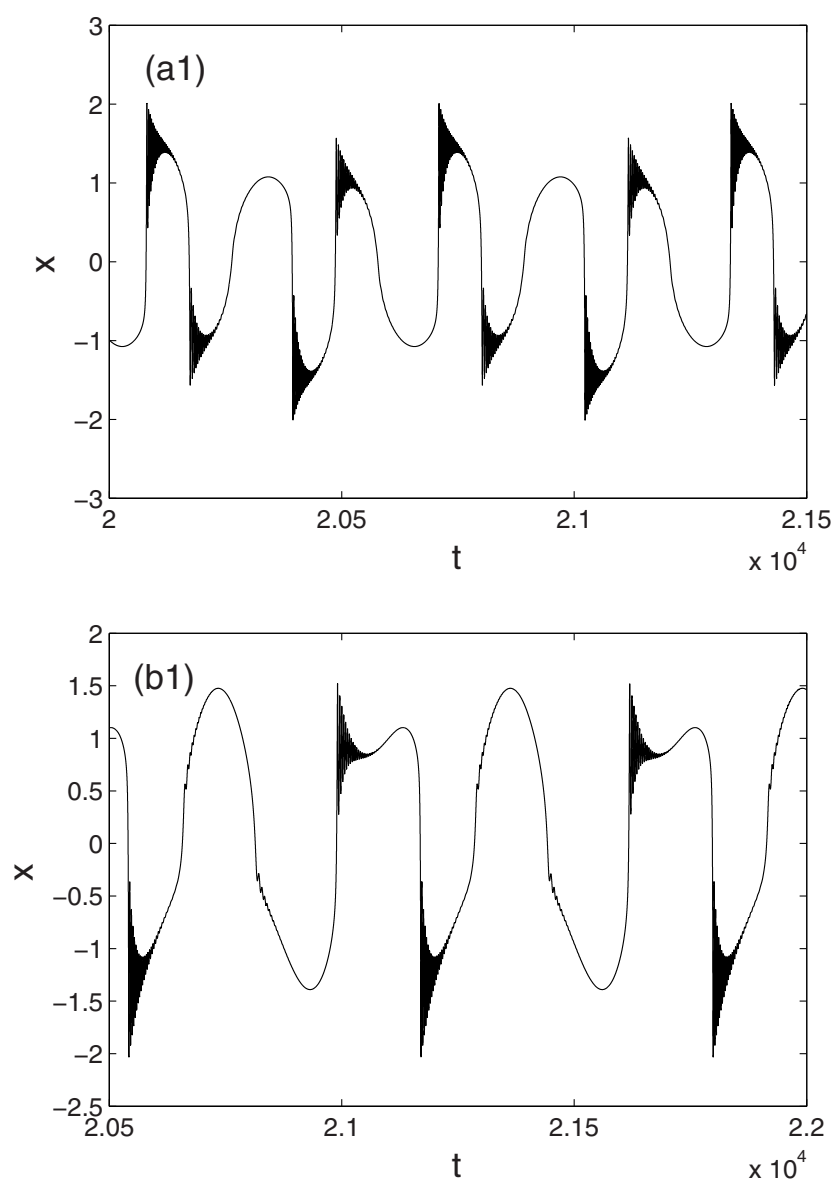

variable, i.e., $g(t)=\cos (0.01 t)$. This leads to the fast subsystem

$$
\ddot{x}+0.1 \dot{x}-(0.5+\gamma) x+x^{3}=\gamma,
$$

where $\gamma=g(t)=\cos (0.01 t)$ is the control parameter (see Table I, row 1), which modulates the dynamics of the fast subsystem. This fast subsystem exhibits an S-shaped equilibrium curve with two fold bifurcations as shown in Fig. 2(a1). In Fig. 2(a2), the related transformed phase diagram [21] of the MMOs is presented and the S-shaped equilibrium curve is also overlayed and reveals the bifurcation mechanism of the MMOs. The numerical simulations in Fig. 2(a2) show reasonable agreement between the transformed phase diagram and the bifurcation diagram, which complements the fact that it is the switchings between the two stable equilibria that form the MMOs in Fig. 1(a).

When $\omega_{1}=0.01$ and $\omega_{2}=0.02$, according to case $\mathrm{C}$, we get $g(t)=\cos (0.01 t), f_{1}(x)=x$, and $f_{2}(x)=f_{2}^{*}(x)=$ $2 x^{2}-1$. This leads to the fast subsystem shown in Table I, row 2 . Since the equilibrium curve of the fast subsystem displays two fold bifurcations [see Fig. 2(b1)], the MMOs in Fig. 1(b) have the same dynamical mechanism as that of the MMOs in Fig. 1(a), i.e., the MMOs are also generated by the switchings between two stable equilibria of the fast subsystem [see Fig. 2(b2)]. However, compared to the S-shaped equilibrium curve in Fig. 2(a1), the equilibrium curve in Fig. 2(b1) has
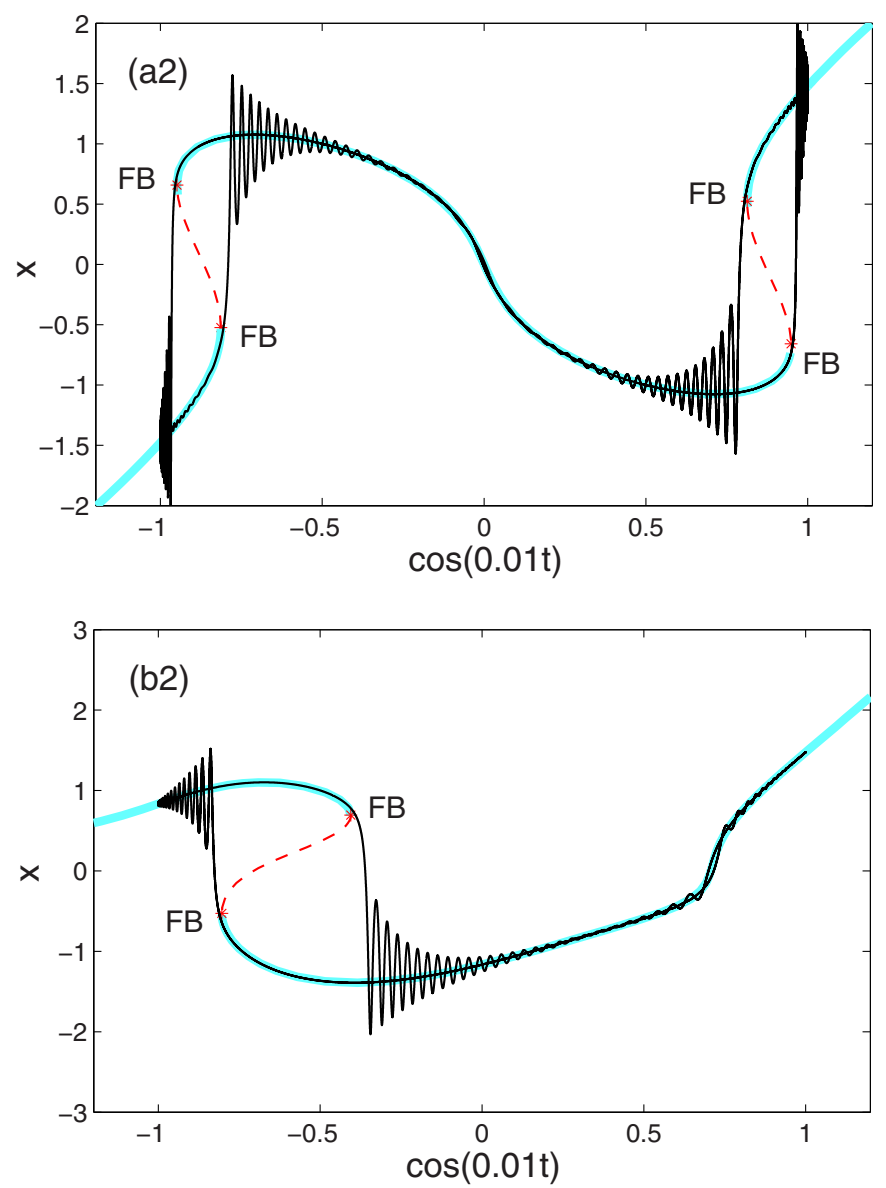

FIG. 6. (Color online) (a1) and (a2) Same as in Fig. 5 for $\omega_{1}=0.02$ and $\omega_{2}=0.03$, with the related fast subsystem shown in Table II, row 4. (b1) and (b2) Same as in Fig. 5 for $\omega_{1}=0.03$ and $\omega_{2}=0.02$. The associated fast subsystem is shown in Table II, row 5. 

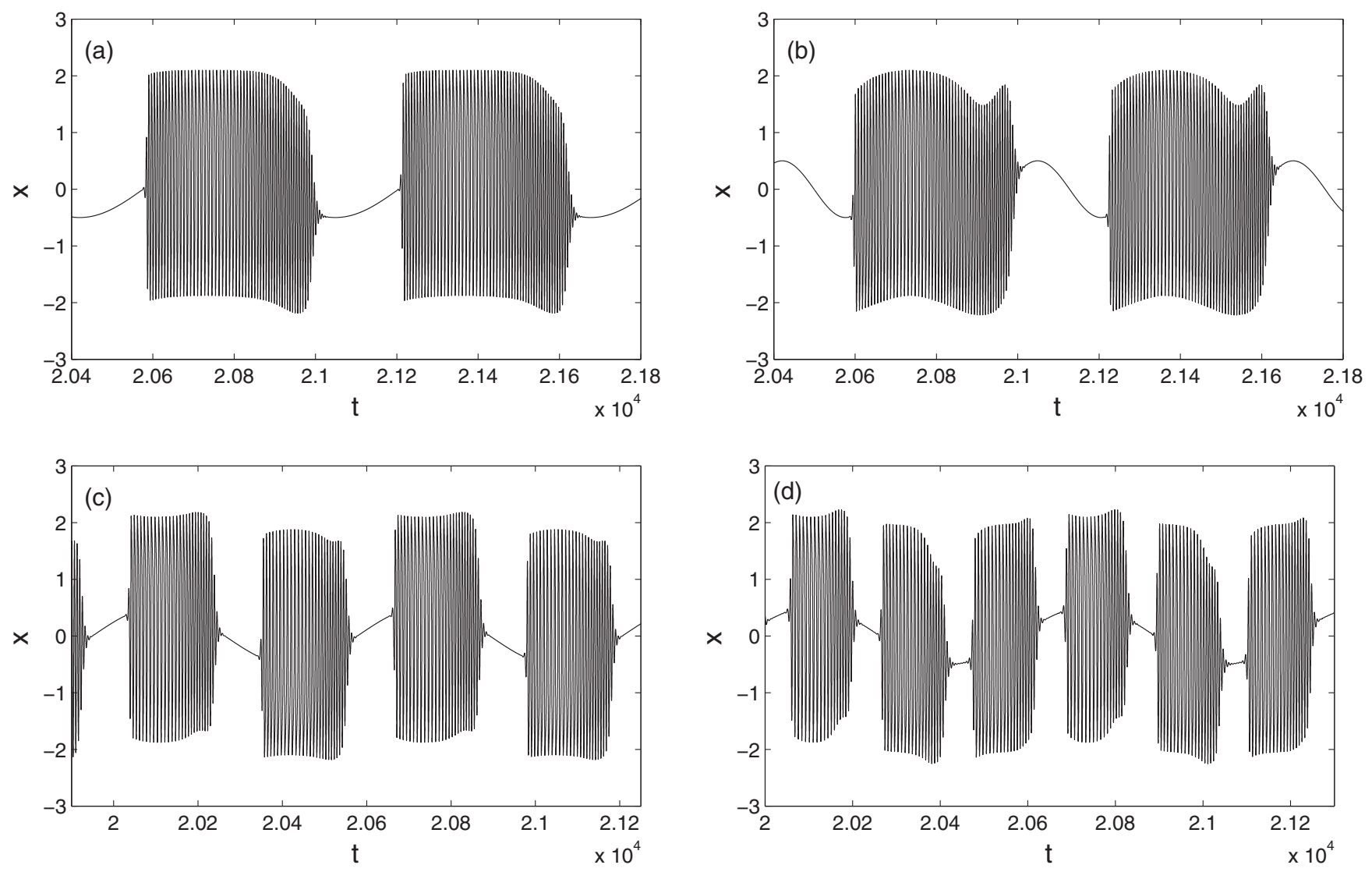

FIG. 7. Mixed-mode oscillations in the van der Pol system (14) for (a) $\omega_{1}=\omega_{2}=0.01$, (b) $\omega_{1}=0.01$ and $\omega_{2}=0.02$, (c) $\omega_{1}=0.02$ and $\omega_{2}=0.01$, and (d) $\omega_{1}=0.03$ and $\omega_{2}=0.01$.

a tortuous lower branch and this answers for the different characteristic observed in the MMOs [see the circled region in Fig. 1(b)].

When $\omega_{1}=0.01$ and $\omega_{2}=0.03$, we have $g(t)=$ $\cos (0.01 t), f_{1}(x)=x$, and $f_{2}(x)=f_{3}^{*}(x)=4 x^{3}-3 x$. This leads to the fast subsystem shown in Table I, row 3. Two additional fold bifurcation points are created by considering $\gamma$ as the control parameter and in all, four fold bifurcation points are obtained [see Fig. 3(a1)]. We overlay the equilibrium curve with the transformed phased diagram of MMOs, which explains the four catastrophic jumps observed in each period of the MMOs [see Figs. 1(c) and 3(a2)].

When $\omega_{1}=0.01$ and $\omega_{2}=0.04$, we have $g(t)=$ $\cos (0.01 t), f_{1}(x)=x$, and $f_{2}(x)=f_{4}^{*}(x)=8 x^{4}-8 x^{2}+1$. Then we obtain the fast subsystem shown in Table I, row 4. For this case, as shown in Fig. 3(b1), six fold bifurcation points are obtained by considering $\gamma=\cos (0.01 t)$ as the control parameter. In Fig. 3(b2), the bifurcation diagram is overlayed with the transformed phase diagram of MMOs, which reveals the six catastrophic jumps observed in each period of the MMOs [see Figs. 1(d) and 3(b2)].

Dynamical mechanisms of the MMOs for the above four cases have been revealed, which in turn demonstrate the effectiveness of the method. In the following two paragraphs, we predict some dynamical behavior of the Duffing system (12). If the method works, it should be able to make predictions on the behavior of the system.

We consider the case when $\omega_{1}=\omega_{2}=\varepsilon$, where $0<\varepsilon \ll 1$ and $\varepsilon \neq 0.01$. Compared to the case when $\omega_{1}=\omega_{2}=0.01$, the two frequencies vary, however, the relation between them remains the same, i.e., $\omega_{1}=\omega_{2}$. According to our method, this leads to the same fast subsystem with the same bifurcation behavior by choosing $g(t)=\cos (\varepsilon t)$ as the slow variable. Therefore, a MMO pattern with the same dynamical mechanism as that of the case when $\omega_{1}=\omega_{2}=0.01$ is created [see Fig. 1(e)]. Similarly, one may conclude that a MMO pattern with the same dynamical mechanism as that of the MMOs in Fig. 1(b) can be obtained for the case when $\omega_{1}=\varepsilon$

TABLE III. Parametrically and externally excited van der Pol equation and the associated fast subsystem with different $\omega_{1}$ and $\omega_{2}$.

\begin{tabular}{lccc}
\hline \hline$\omega_{1}$ & $\omega_{2}$ & Parametrically and externally excited van der Pol equation & Fast subsystem \\
\hline 0.01 & 0.01 & $\ddot{x}+[1+1.5 \cos (0.01 t)]\left(x^{2}-1\right) \dot{x}+x=0.5 \cos (0.01 t)$ & $\ddot{x}+(1+1.5 \gamma)\left(x^{2}-1\right) \dot{x}+x=0.5 \gamma$ \\
0.01 & 0.02 & $\ddot{x}+[1+1.5 \cos (0.01 t)]\left(x^{2}-1\right) \dot{x}+x=0.5 \cos (0.02 t)$ & $\ddot{x}+(1+1.5 \gamma)\left(x^{2}-1\right) \dot{x}+x=0.5\left(2 \gamma^{2}-1\right)$ \\
0.02 & 0.01 & $\ddot{x}+[1+1.5 \cos (0.02 t)]\left(x^{2}-1\right) \dot{x}+x=0.5 \cos (0.01 t)$ & $\ddot{x}+\left[1+1.5\left(2 \gamma^{2}-1\right)\right]\left(x^{2}-1\right) \dot{x}+x=0.5 \gamma$ \\
0.03 & 0.01 & $\ddot{x}+[1+1.5 \cos (0.03 t)]\left(x^{2}-1\right) \dot{x}+x=0.5 \cos (0.01 t)$ & $\ddot{x}+\left[1+1.5\left(4 \gamma^{3}-3 \gamma\right)\right]\left(x^{2}-1\right) \dot{x}+x=0.5 \gamma$ \\
\hline \hline
\end{tabular}


and $\omega_{2}=2 \varepsilon$, where $0<\varepsilon \ll 1$ and $\varepsilon \neq 0.01$ [see Fig. 1(f) for example], since for this case the slow variable can be chosen as $g(t)=\cos (\varepsilon t)$ and the resulting fast subsystem and its bifurcation behavior have not changed.

When $\omega_{1}=0.02,0.03$, and $0.04\left(\omega_{2}\right.$ is fixed at $\left.\omega_{2}=0.01\right)$, the parametrically and externally excited Duffing equations are listed in Table II, rows 1-3, respectively, where the associated fast subsystems are also given by our method based on case B. By considering $\gamma$ as the control parameter of the fast subsystems, the associated equilibrium curves are plotted in Fig. 4. When $\omega_{1}=0.02$, as shown in Fig. 4(a), there is no bifurcation on the equilibrium curve, which indicates that no MMOs will be created [see Figs. 5(a1) and 5(a2)]. However, when $\omega_{1}=0.03$, the equilibrium curves become flexural and two fold bifurcation points are created. Therefore, qualitatively, a MMOs pattern, which has the same dynamical mechanism as that of the MMOs pattern in Fig. 1(a), can be created when the slow variable $\cos (0.01 t)$ periodically passes through the two fold bifurcation points [see Figs. 5(b1) and 5(b2)]. Similarly, a MMO pattern, which has the same dynamical mechanism, will be created for the case when
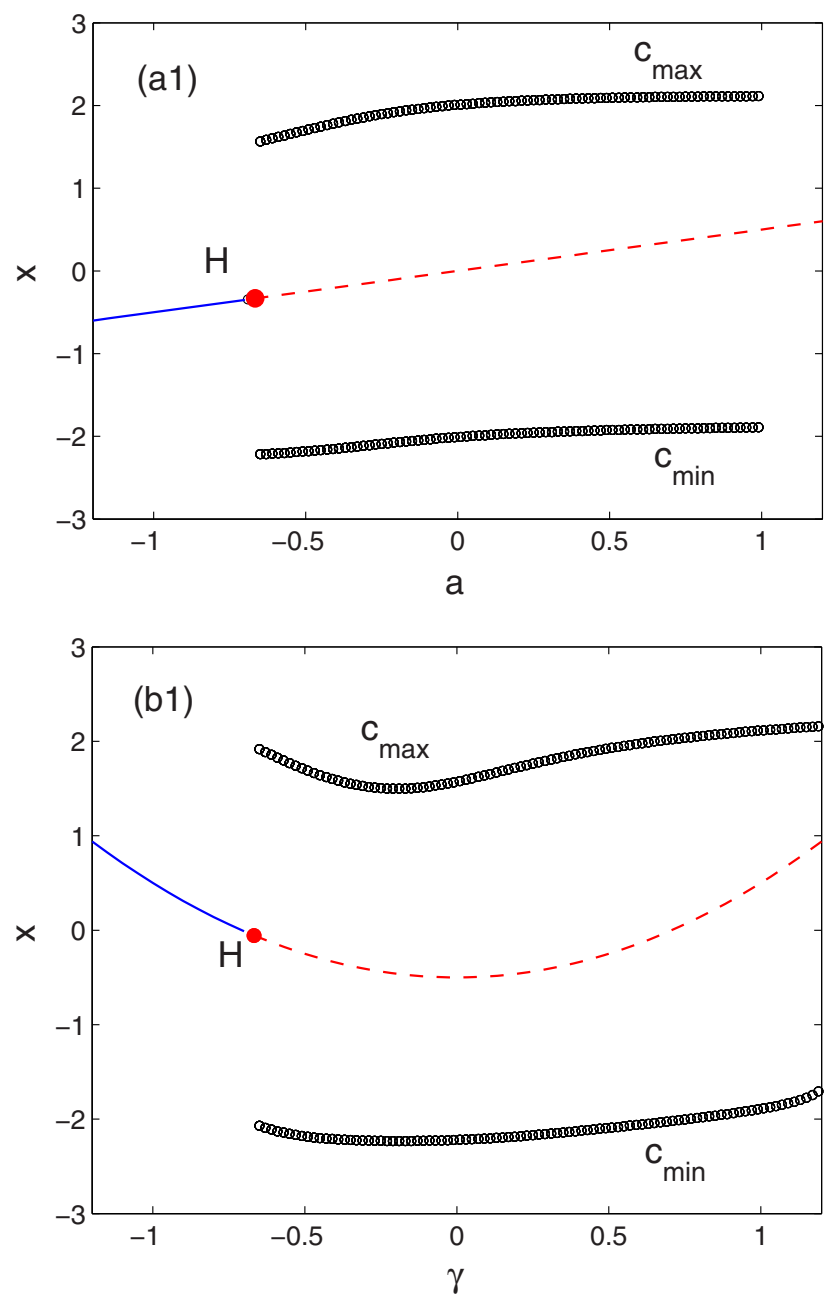

$\omega_{1}=0.04$ since the equilibrium curve also displays two fold bifurcations [see Figs. 4(c), 5(c1), and 5(c2)].

Finally, we conclude this section by discussing two examples related to case A. When $\omega_{1}=0.02$ and $\omega_{2}=0.03$, a MMO pattern is exhibited in Fig. 6(a1). According to case A, we have $g(t)=\cos (0.01 t), f_{1}(x)=f_{2}^{*}(x)=2 x^{2}-1$, and $f_{2}(x)=f_{3}^{*}(x)=4 x^{3}-3 x$, which gives rise to the fast subsystem in Table II, row 4. Further numerical results show that the MMO pattern in Fig. 6(a1) has the same dynamical mechanism as that of the MMO pattern in Fig. 1(c) since their respective fast subsystems exhibit the same bifurcation modes [see Figs. 3(a2) and 6(a2)]. When $\omega_{1}=0.03$ and $\omega_{2}=0.02$, the method reveals that a MMO pattern, which has the same dynamical mechanism as that of the case when $\omega_{1}=0.01$ and $\omega_{2}=0.02$, is exhibited [see Figs. 2(b1), 2(b2), 6(b1), and 6(b2)].

\section{B. van der Pol equation}

We consider the following parametrically and externally excited van der Pol equation with two low excitation frequencies,
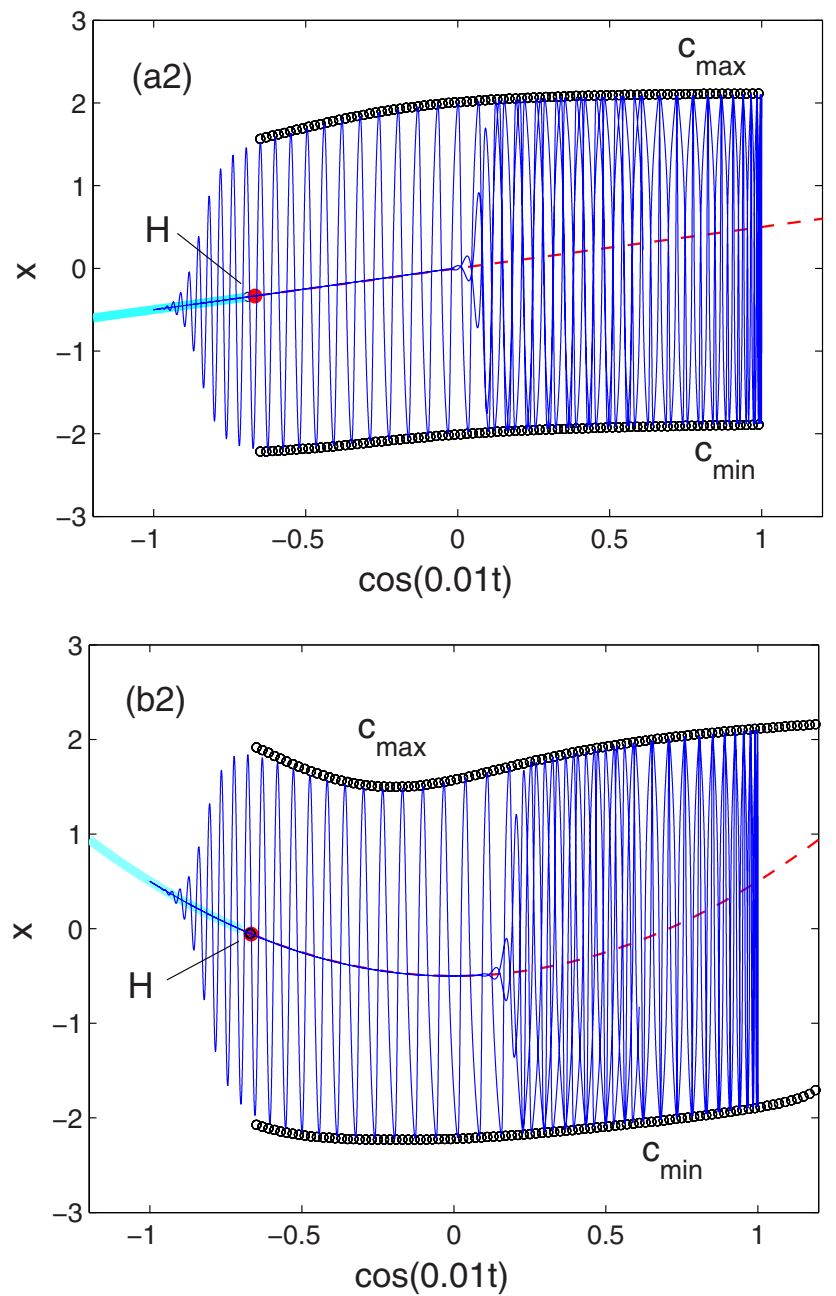

FIG. 8. (Color online) (a1) Bifurcation diagram of the fast subsystem for $\omega_{1}=\omega_{2}=0.01$, where $\gamma$ is used as the control parameter: solid (dashed) curve, stable (unstable) equilibrium; $H$, supercritical Hopf bifurcation; and $c_{\max }$ and $c_{\min }$, the maximum and minimum amplitudes of stable oscillations generated from $H$. (a2) Overlay of the bifurcation diagram with the transformed phase diagram of MMOs, where the stable equilibrium is highlighted with thick blue curves. (b1) and (b2) Same as in (a1) and (a2) for $\omega_{1}=0.01$ and $\omega_{2}=0.02$. 
given by

$$
\ddot{x}+\left[1+\beta_{1} \cos \left(\omega_{1} t\right)\right]\left(x^{2}-1\right) \dot{x}+x=\beta_{2} \cos \left(\omega_{2} t\right),
$$

where $0<\omega_{1,2} \ll 1, \beta_{1}=1.5, \beta_{2}=0.5$, and the frequencies $\omega_{1,2}$ are the control parameters. When $\omega_{1}=\omega_{2}=0.01$, a MMO pattern is obtained, which shows one cluster of repetitive spiking during each period of MMO [see Fig. 7(a)]. To uncover the dynamical mechanisms underlying the appearance of such
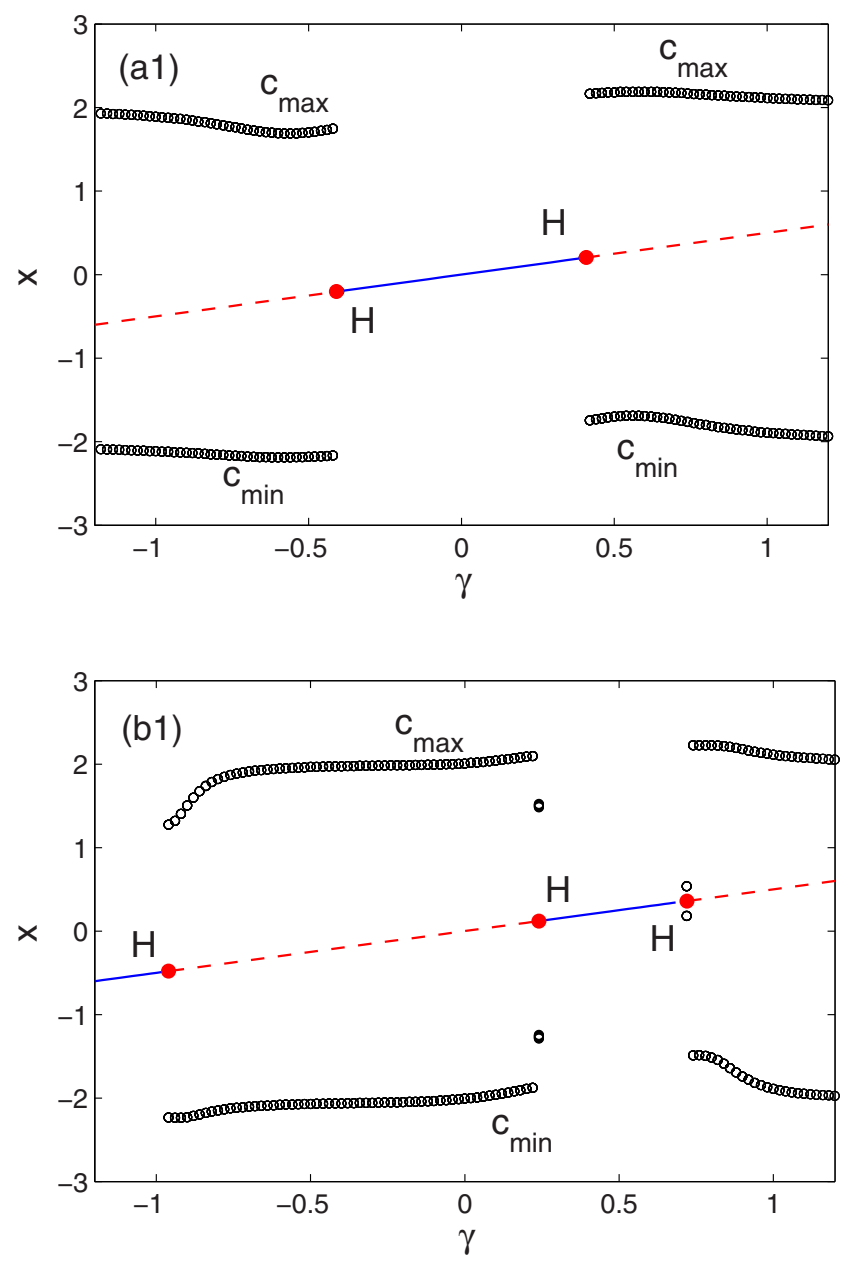

MMOs, we first consider the excitation $\cos (0.01 t)$ as the slow variable and then list the fast-slow system and its subsystem in Table III, row 1. Figure 8(a1) shows that the fast subsystem displays a supercritical Hopf bifurcation. In Fig. 8(a2), the transformed phase diagram is also superimposed, which indicates the fact that the MMOs are formed by periodic switchings between an equilibrium attractor and a limit cycle attractor, while such switchings are modulated by supercritical Hopf
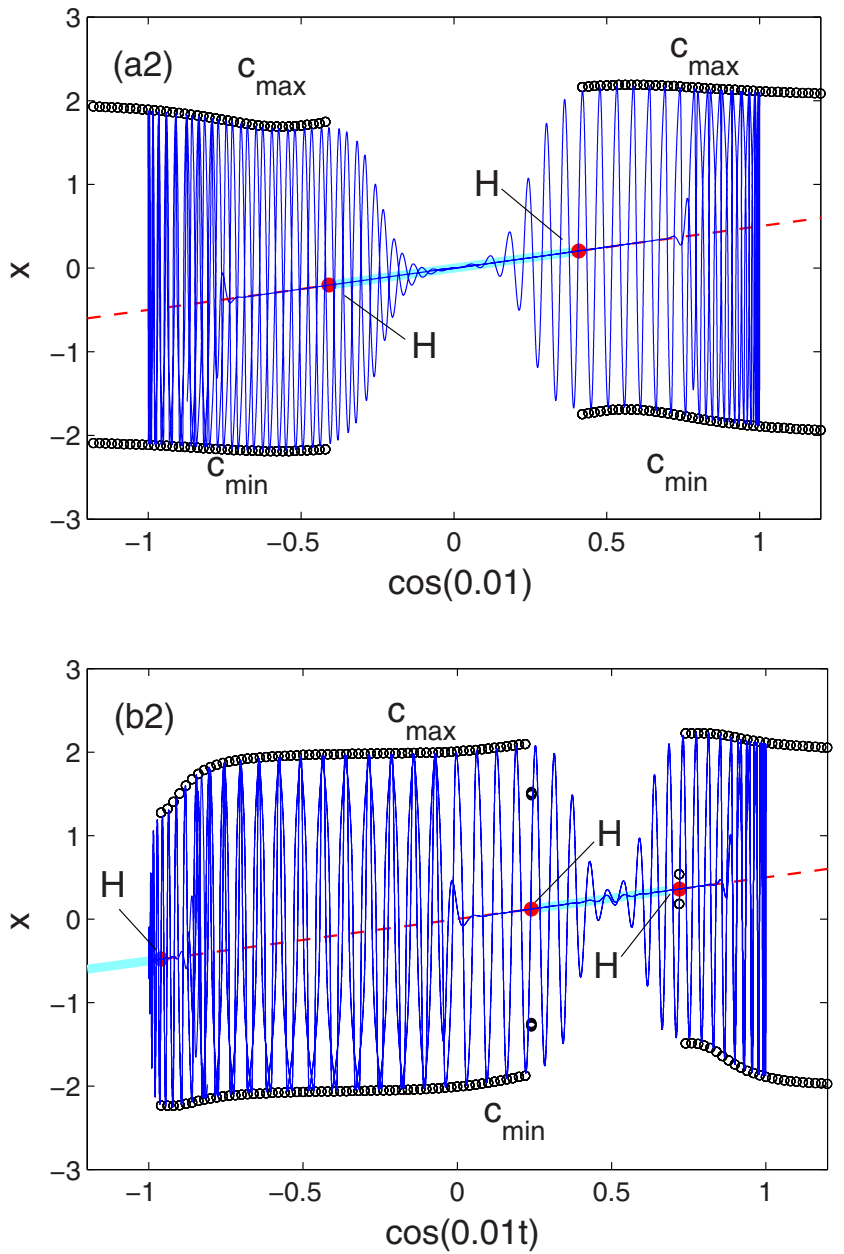

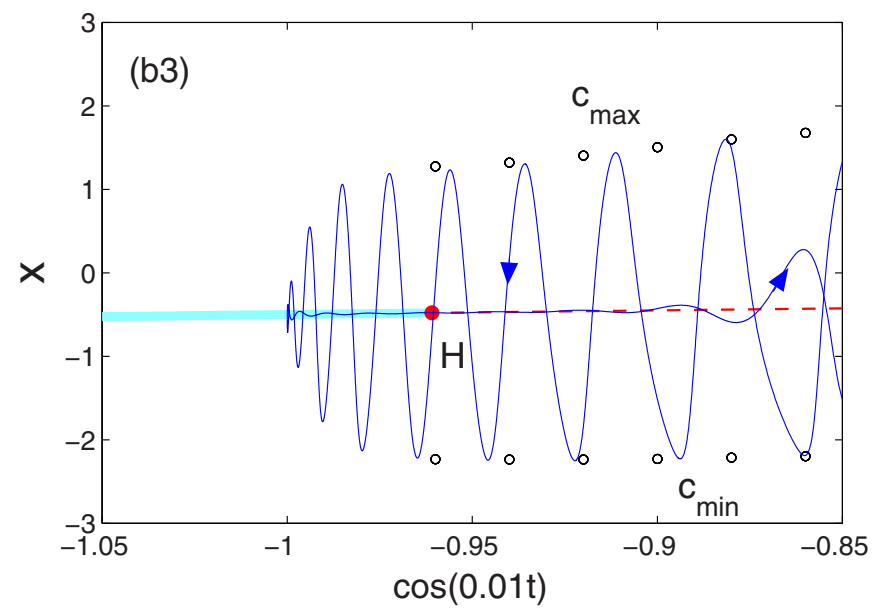

FIG. 9. (Color online) (a1) and (a2) Same as in Fig. 8 for $\omega_{1}=0.02$ and $\omega_{2}=0.01$ and (b1)-(b3) same as in Fig. 8 for $\omega_{1}=0.03$ and $\omega_{2}=0.01$, with (b3) the enlargement of (b2). 
bifurcations when the slow variable $\cos (0.01 t)$ periodically passes through the Hopf bifurcation point $H$.

When $\omega_{1}=0.01$ and $\omega_{2}=0.02$, a similar periodic MMO pattern can be observed in Fig. 7(b). By choosing $\cos (0.01 t)$ as the slow variable and using the method proposed, the fast-slow system and its fast subsystem are listed in Table III, row 2. The same dynamical mechanism as that of the MMO pattern in Fig. 7(a) is demonstrated by the fast-slow analysis shown in Figs. 8(b1) and 8(b2).

However, when $\omega_{1}=0.02$ and $\omega_{2}=0.01$, the MMOs show some essential differences, i.e., two clusters of repetitive spiking during each period of MMO occur now [see Fig. 7(c)]. The generation of such MMOs can be well understood [see Table III, row 3 and Figs. 9(a1) and 9(a2)]. The fast subsystem displays two supercritical Hopf bifurcations (resulting from the changing of the fast subsystem), which bound the region of an equilibrium attractor and outside, which are two limit cycle attractors. When the slow variable $\cos (0.01 t)$ is switched on, the system undergoes a transition from the equilibrium to the two limit cycles, which leads to a MMO pattern with two clusters of repetitive spiking during each period. When $\omega_{1}=0.03$ and $\omega_{2}=0.01$, a third supercritical Hopf bifurcation point is created, which leads to a MMO pattern with three clusters of repetitive spiking during each period [see Fig. 7(d); Table III, row 4; and Figs. 9(b1)-9(b3)].

\section{DISCUSSION}

Mixed-mode oscillations are a complex, multiple-timescale dynamical behavior and the deep understanding of such oscillations is important. In this paper we have proposed a framework to study MMOs in PEESTLEFs for the case when the two slow excitation frequencies are rationally related by introducing a slow variable and finding the relation between such a variable and the slow excitations. Mixed-mode oscillations can be understood by the study of the transformed fast-slow system with a single slow variable. Our method has explained the occurrence of MMOs: MMOs are generated when the slow variable periodically passes through bifurcation points of the fast subsystem of the transformed fast-slow system. We have also investigated the mechanisms underlying the appearance of different MMOs: The fast subsystem of the transformed fast-slow system changes along with the two excitation frequencies, which often leads to different bifurcation modes, and MMOs with different patterns are thus created.

According to the classical fast-slow analysis, MMOs occur since the trajectory undergoes a switching between attractors of the fast subsystem; such switching is modulated by the slow variable when it periodically passes through bifurcation points of the subsystem. Therefore, MMOs have the same frequency as that of the slow variable. With respect to PEESTLEFs, since they can be transformed into the classical fast-slow forms with a single slow variable $g(t)$, one may conclude that MMOs observed in PEESTLEFs have the same frequency as that of $g(t)$ (see Fig. 6 for an example). On the other hand, we have shown that if one of the two frequencies of excitations is a multiple of the other one (see cases B and C), the excitation with the lower frequency can be chosen as the slow variable
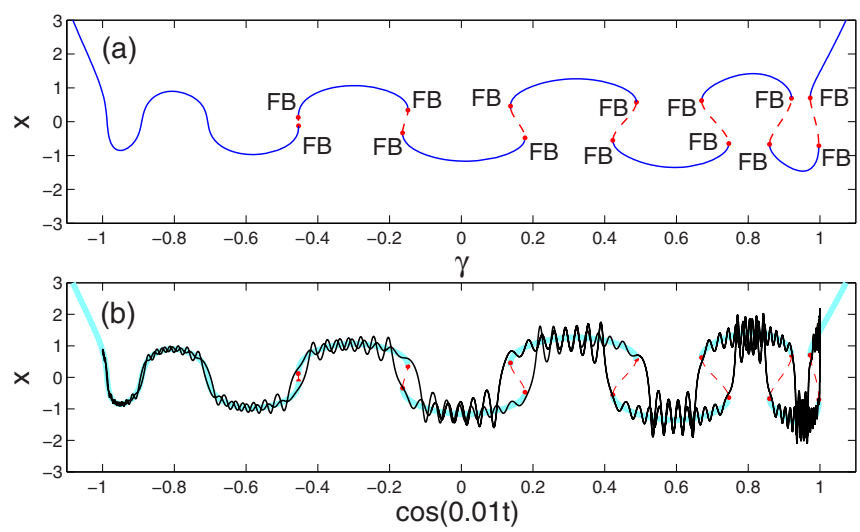

FIG. 10. (Color online) Complex periodic MMO pattern and its dynamical mechanism in the system (12) for $\omega_{1}=0.01$ and $\omega_{2}=0.1$. Since $\cos (0.1 t)=f_{10}^{*}(\cos (0.01 t))=\left[2 \cos ^{2}(0.01 t)-\right.$ $1]\left[256 \cos ^{8}(0.01 t)-512 \cos ^{6}(0.01 t)+304 \cos ^{4}(0.01 t)-48 \cos ^{2}\right.$ $(0.01 t)+1]$, we have $g(t)=\cos (0.01 t), f_{1}(x)=x$, and $f_{2}(x)=$ $\left(2 x^{2}-1\right)\left(256 x^{8}-512 x^{6}+304 x^{4}-48 x^{2}+1\right)$, which leads to the fast subsystem $\ddot{x}+0.1 \dot{x}-(0.5+\gamma) x+x^{3}=\left(2 \gamma^{2}-1\right)\left(256 \gamma^{8}-\right.$ $\left.512 \gamma^{6}+304 \gamma^{4}-48 \gamma^{2}+1\right)$, where $\gamma$ is the control parameter. (a) Bifurcation diagram of the fast subsystem, where $\gamma$ is the control parameter. The FB denote fold bifurcations of equilibria. (b) Overlay of the bifurcation diagram with the transformed phase diagram of the complex MMOs, where the stable branches of equilibrium are highlighted.

$g(t)$. So, in such two cases, the MMOs have the same frequency as that of the excitation with the lower frequency (see Figs. 2, $3,5,8$, and 9 for examples).

We would like to point out that complex MMOs are common in PEESTLEFs. This is not surprising since complex bifurcation behavior can be generated when the excitation frequencies vary continually. For example, as shown in Fig. 10(a), the equilibrium curve of the fast subsystem shows more twists and turns and complements the generation of complex periodic MMOs [see Figs. 10(b) and 11(a)]. With the multiplication of the frequency, the resulting bifurcation
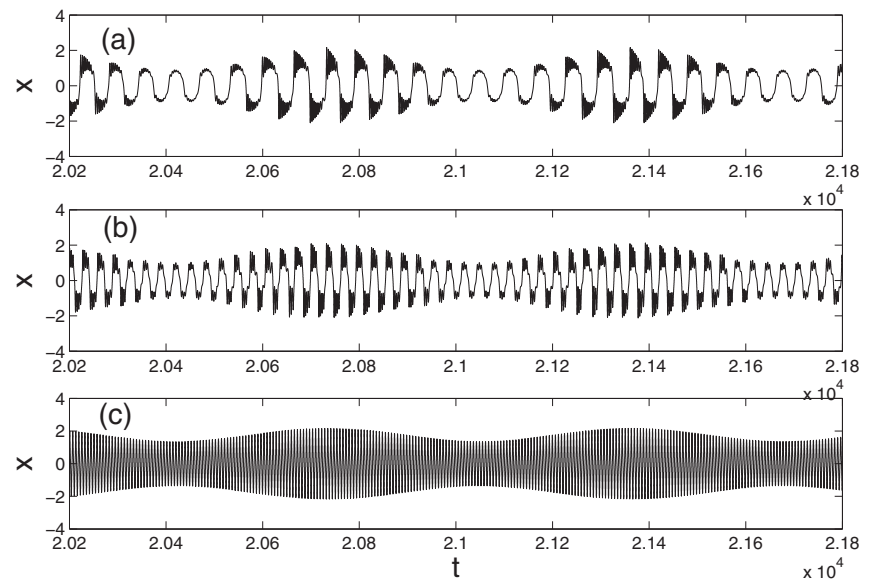

FIG. 11. Transition from (a) and (b) complex periodic MMOs to (c) quasiperiodic oscillations for (a) $\omega_{1}=0.01$ and $\omega_{2}=0.1$ [time series of the MMOs in Fig. 10(b)], (b) $\omega_{1}=0.01$ and $\omega_{2}=0.2$, and (c) $\omega_{1}=0.01$ and $\omega_{2}=1$. 
behavior becomes far more complicated and finally such complex periodic MMOs evolve to nonperiodic oscillations (see Fig. 11).

Even though we give an explanation for the generation of MMOs, we do not consider the case when the two excitation frequencies are incommensurate, since in this case it is difficult to determine the slow variable $g(t)$. However, numerical simulations show that MMOs, especially complex nonperiodic MMOs are ubiquitous for the incommensurate case and further work is needed on this topic.

\section{ACKNOWLEDGMENTS}

The authors express their gratitude to the anonymous reviewers for their valuable comments and suggestions that help to improve the paper. This work was supported by the National Natural Science Foundation of China (Grants No. 11202085, No. 21276115, No. 11302087, No. 11302086, and No. 11402226), the Natural Science Foundation of Jiangsu Province (Grant No. BK20130479), and the Research Foundation for Advanced Talents of Jiangsu University (Grant No. 11JDG075 ).
[1] D. Gapinski, Z. Koruba, and I. Krzysztofik, Mech. Syst. Signal Process. 45, 433 (2014).

[2] J. R. Hervas and M. Reyhanoglu, Acta Astronaut. 98, 120 (2014).

[3] I. Elizalde and J. Ancheyta, Fuel 138, 45 (2014).

[4] M. Farsi and A. Jahanmiri, J. Ind. Eng. Chem. 20, 2927 (2014).

[5] S. M. Baer, T. Erneux, and J. Rinzel, SIAM J. Appl. Math. 49, 55 (1989).

[6] L. Holden and T. Erneux, SIAM J. Appl. Math. 53, 1045 (1993).

[7] D. C. Diminnie and R. Haberman, J. Nonlinear Sci. 10, 197 (2000).

[8] A. N. Pisarchik, V. N. Chizhevsky, R. Corbalán, and R. Vilaseca, Phys. Rev. E 55, 2455 (1997).

[9] A. N. Pisarchik, B. F. Kuntsevich and R. Corbalán, Phys. Rev. E 57, 4046 (1998).

[10] A. N. Pisarchik and B. K. Goswami, Phys. Rev. Lett. 84, 1423 (2000).

[11] X. J. Han, Q. S. Bi, C. Zhang, and Y. Yu, Int. J. Bifurcat. Chaos 24, 1450098 (2014)

[12] J. Gukenheimer and P. Holmes, Nonlinear Oscillations, Dynamical Systems and Bifurcation of Vector Field (Springer, New York, 1983).

[13] J. Grasman, Asymptotic Methods for Relaxation Oscillations and Applications (Springer, New York, 1987).

[14] S. Wiggins, Global Bifurcation and Chaos: Analytical Methods (Springer, New York, 1988).

[15] S. Wiggins, Introduction to Applied Nonlinear Dynamical Systems and Chaos (Springer, New York, 1990).

[16] A. H. Nayfeh, Introduction to Perturbation Techniques (Wiley, New York, 1993).

[17] A. H. Nayfeh and D. T. Mook, Nonlinear Oscillations (Wiley, New York, 1995).

[18] K. T. Alligood, T. D. Sauer, and J. A. Yorke, CHAOS: An Introduction to Dynamical Systems (Springer, New York, 1996).

[19] M. Golubitsky, K. Josic, and T. J. Kaper, in Global Analysis of Dynamical Systems: Festschrift Dedicated to Floris Takens on the Occasion of his 60th Birthday, edited by H. W. Broer, B. Krauskopf, and G. Vegter (Institute of Physics, Bristol, 2001), pp. 277-308.

[20] R. Bertram, M. J. Butte, T. Kiemel, and A. Sherman, Bull. Math. Biol. 57, 413 (1995).
[21] X. J. Han and Q. S. Bi, Commun. Nonlinear Sci. Numer. Simul. 16, 4146 (2011).

[22] X. J. Han and Q. S. Bi, Nonlinear Dyn. 68, 275 (2012).

[23] X. J. Han and Q. S. Bi, Sci. China Technol. Sci. 55, 702 (2012).

[24] E. M. Izhikevich, Int. J. Bifurcat. Chaos. 10, 1171 (2000).

[25] Q. S. Lu, H. G. Gu, Z. Q. Yang, X. Shi, L. X. Duan, and Y. H. Zheng, Acta Mech. Sin. 24, 593 (2008).

[26] J. Rinzel, in Ordinary and Partial Differential Equations, edited by B. D. Sleeman and R. J. Jarvis (Springer, Berlin, 1985), pp. 304-316.

[27] L. X. Duan, Q. S. Lu, and Q. Y. Wang, Neurocomputing 72, 341 (2008).

[28] Z. Q. Yang, Q. S. Lu, and L. Li, Chaos Soliton. Fract. 27, 87 (2006).

[29] R. Straube, D. Flockerzi, and M. J. B. Hauser, J. Phys.: Conf. Ser. 55, 214 (2006)

[30] A. H. Abbasian, H. Fallah, and M. R. Razvan, Biol. Cybern. 107, 465 (2013).

[31] J. Rinzel, in Mathematical Topics in Population Biology, Morphogenesis and Neurosciences, edited by E. Teramoto and M. Yamaguti, Lecture Notes in Biomathematics Vol. 71 (Springer, Berlin, 1987), pp. 267-281.

[32] W. B. Adams and J. A. Benson, Prog. Biophys. Mol. Biol. 46, 1 (1985).

[33] A. Milik, P. Szmolyan, H. Löffelmann, and E. Gröller, Int. J. Bifurcat. Chaos 8, 505 (1998).

[34] M. Krupa, A. Vidal, M. Desroches, and F. Clément, SIAM J. Appl. Dyn. Syst. 11, 1458 (2012).

[35] R. Curtu, Physica D 239, 504 (2010).

[36] J. Guckenheimer, SIAM J. Appl. Dyn. Syst. 7, 1355 (2008).

[37] M. Watts, J. Tabak, C. Zimliki, A. Sherman, and R. Bertram, J. Theor. Biol. 276, 218 (2011).

[38] X. H. Li and Q. S. Bi, Chinese Phys. B 21, 060505 (2012).

[39] M. Desroches, J. Guckenheimer, B. Krauskopf, C. Kuehn, H. M. Osinga, and M. Wechselberger, SIAM Rev. 54, 211 (2012).

[40] H. Michiel, De Moivre Formula, Encyclopedia of Mathematics (Springer, New York, 2001).

[41] Y. Q. Tang, L. Q. Chen, and X. D. Yang, J. Sound Vib. 320, 1078 (2009).

[42] G. Litak, A. Syta, and M. Borowiec, Chaos Soliton. Fract. 32, 694 (2007). 